\title{
Systematic errors in the evaluation of uncorrected data from thermographic lock-in measurements
}

by R. Krankenhagen, M. Ziegler and C. Maierhofer

Bundesanstalt für Materialforschung und -prüfung (BAM), Division 8.7: Thermographic Methods, Unter den Eichen 87, 12205 Berlin, Germany, rainer.krankenhagen@bam.de

\begin{abstract}
Lock-in thermography (LT) is based on the correct evaluation of phase differences between the temperature oscillations at different surface regions of the object under test during periodic heating. Since the usual heating procedures contain a DC component, the actual heating pattern achieved is not harmonic. This causes systematic deviations when phase differences are determined by means of harmonic analysis, e.g. with FFT analysis. The resulting errors depend clearly on the ratio between DC and AC amplitude, which is demonstrated at simulated and experimentally recorded temperature transients. Further experimental LT data obtained by different oscillating energy inputs showed a variety of possible shapes of transients with different DC components.
\end{abstract}

\section{Introduction}

Lock-in thermography (LT) is an established non-destructive test method in which the surface of the sample is subjected to periodic heating. The basics are long known (e. g. [1] from 1992). Subsurface structural features or defects within a solid lead to a changed heat flow within the sample, which in turn influences the surface temperature at the corresponding points. When the surface is periodically heated, a temperature oscillation occurs at these points with a phase shift in relation to undisturbed areas. This phase difference represents the actual useful signal. Under certain assumptions, the value of the phase difference can be used to deduce a statement of defect depth [2]. With the transition from single detector cameras to focal plane array detectors conventional lock-in amplifiers could no longer be used. Instead the periodic behavior of temperature transients of every pixel has to be analyzed digitally. This is realized during the measurement after every single period (online-mode) as well as after the complete measurement. The online-mode requires special software that performs the necessary calculations during runtime (section 3.2 in [3]). Alternatively, special signal electronics can be used if a phase-locked temperature signal is available during runtime [4]. Data evaluation at the end of the measurement is usually realized offline by a Fast Fourier Transformation (FFT) or Discrete Fourier Transformation of multiples of an oscillation period (section 2.2 in [3].

In optically excited lock-in thermography, the induced heat flux density at the sample surface $\boldsymbol{q}$ is given by

$$
q(t)=\frac{a}{2} I_{0}\left[1+\sin \left(2 \pi f t-\frac{\pi}{2}\right)\right]
$$

( $\boldsymbol{a}$ absorptance of the sample surface at light source wavelength, $\boldsymbol{I}_{\mathbf{0}}$ amplitude of irradiance of light source, $\boldsymbol{f}$ modulation frequency, $\boldsymbol{t}$ time). The phase shift of $\frac{\pi}{2}$ in (1) is used to let the heating start with $\boldsymbol{q}(\boldsymbol{t}=\mathbf{0})=\mathbf{0}$. Hence, the temperature oscillation is only generated by periodic heating (i.e., the AC component given by the Sine in brackets), whereas the sample continues to heat up over many periods (i.e., the DC component given by the 1 in brackets). The general temperature transient due to this DC component is a linear temperature rise determined by the integrated absorbed power and heat capacity of the sample. At higher temperatures thermal losses become more and more significant and reduce the further temperature increase until thermal equilibrium between absorbed power and thermal losses is reached.

The usual photothermal approach is based solely on this AC part, treating it as a source of harmonic thermal waves [5]. But, if correlation of the temperature response of the surface is realized via harmonic functions (e. g. Sine and Cosine) or via FFT, any kind of DC component leads to deviations in the corresponding amplitude and phase values describing the correlation. The operator usually tries to minimize this DC heating effect by a longer heating time until the DC heating and heat losses due to convection and radiation are similar and the slope of the DC component reaches zero [6] [7].

For reasons of economic efficiency, however, the measuring time per sample should be as short as possible. Another reason to deal with the influence of the DC component is the enormous irradiance which is now available through modern laser arrays. This allows very high heat flux densities to be generated which can no longer be compensated by natural losses. Several techniques have been applied to compensate this influence [3]. Breitenstein et. al. showed in an analytical calculation that the influence of any DC component is only determined by the magnitude of the resulting 
temperature change [8] and suggested a simple linear correction. Following this concept Pickering and Almond performed a simple linear fit of only the first two temperature maxima at the beginning to correct the influence of the DC component [9]. However, most of publications dealing with LT contain no statements at all as to whether and how the DC components were corrected e.g. [10-13]. This is particularly critical when calibrations are related to phase angles [14] or the absolute value of a phase difference is required for further calculations [11]. Other authors described their DC component correction careful $[15,16]$.

The aim of this paper is to quantify the disturbing influence of the DC component at LT on phase angles results in order to sensitize the community to the issue. This includes analytical calculated curves as well as measured temperature transients. The measured curves demonstrate the variety of temperature transients under different experimental conditions. An example for uncorrected data shows that the magnitude of the phase error for experimental data roughly corresponds to the value of the analytical calculation.

\section{Simulated temperature transients}

In principle, a constant heat flux density results in a temperature curve proportional to the square root of time or linear to time, depending on whether the sample is thermally thick or thermally thin [3]. Here, this distinction refers to the entire measuring period, i.e. the temperatures at front and back of the sample quickly balance each other out (thin) or a clear temperature gradient remains during the entire measurement period (thick). In addition, heat losses due to convection and radiation influence the temperature transients. Liu et.al. described the temperature transient in terms of a charging curve of a capacitor with a thermal capacitance and a thermal resistance that summarizes the thermal losses [17]. To ease the problem here, the temperature change at a surface $S(t)$ is assumed as a superposition of a sinusoidal curve with amplitude $A_{A C}$ and a linear rise in temperature per period $A_{D C}$. The constant bias (correcting the lack of cooling processes at the beginning) is neglected as well as the initial phase lag of $\pi / 2$.

$$
S(t)=A_{A C} \sin (2 \pi f \cdot t)+A_{D C} f \cdot t
$$

For simplicity, the amplitude $A_{A C}$ is set to $1 \mathrm{~K}$. The first figure 1 illustrates the influence of the parameter $A_{D C}$ on the curve shape of $S(t)$ for $f=50 \mathrm{mHz}$.

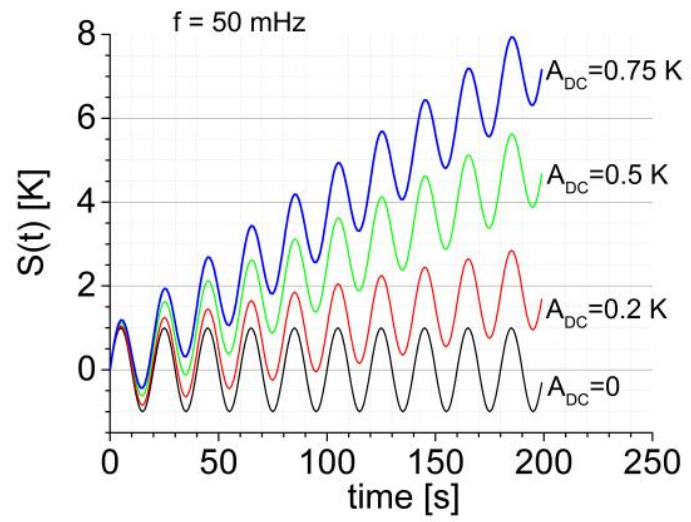

Fig. 1: Influence of the parameter $A_{D C}$ on the curve shape of $S(t)$ according to Eq. (2) for a frequency of $50 \mathrm{mHz}$

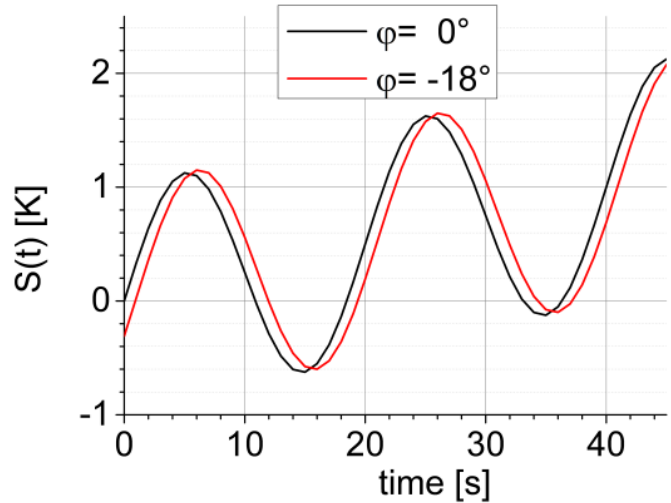

Fig. 2: Influence of a phase lag $\varphi=-18^{\circ}$ on the curve shape of $S(t)$ according to Eq. (3) with $A_{D C}=0.5 \mathrm{~K}$ at a frequency of $50 \mathrm{mHz}$

The next step is the introduction of a relative phase $\varphi$ in order to study the effect of a heat source with delay to the correlation signal (i.e., a phase lag).

$$
S(t)=A_{A C} \sin (2 \pi f \cdot t+\varphi)+A_{D C} f \cdot t
$$


Figure 2 shows two calculated oscillations, each with the same linear rise. The phase difference $\Delta \phi$ between the pure oscillations is exactly $\boldsymbol{\pi} / \mathbf{1 0}$ or $18^{\circ}$. These curves could represent different surface temperature transients of an LT experiment.

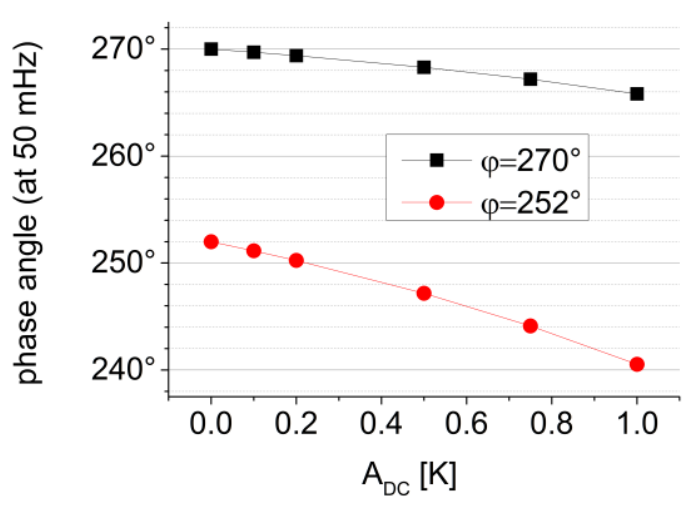

Fig. 3: Influence of the parameter $A_{D C}$ on the resulting phase values (obtained by FFT) for curves with different phase angles according to Eq. (3) at a frequency of $50 \mathrm{mHz}$

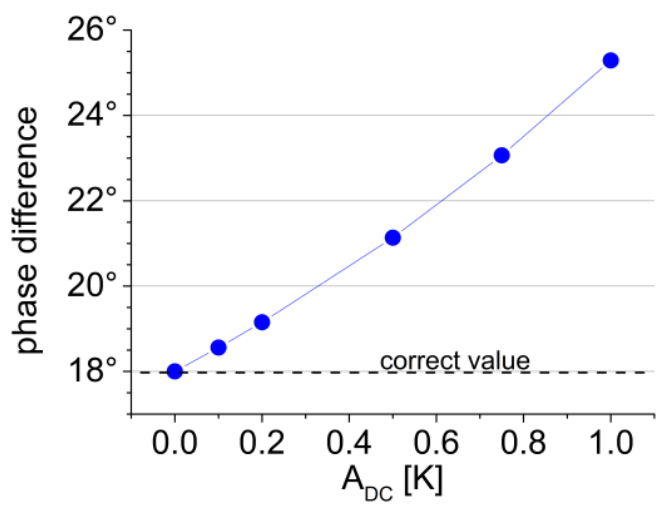

Fig. 4: Influence of the parameter $A_{D C}$ on the resulting phase differences (obtained by FFT) for curves with different phase angles according to Eq. (3) at a frequency of $50 \mathrm{mHz}$

Now, the phase difference between both curves is evaluated just like in a LT measurement. This is carried out by means of a FFT analysis. All FFTs in this contribution were performed with the software Origin 9 from OriginLab Corporation. The obtained phase values are compiled in figure 3 for different DC components described by different $A_{D C}$. The $\varphi$-value of $270^{\circ}$ is a result of the FFT procedure, where real functions are correlated with Cosine.

Although the corresponding transient DC components are exactly the same for both, the original and the phaseshifted signal, the phase values and, more important, their differences change with different DC parts (see figure 4). As to be expected, the magnitude of the deviation from the true $\Delta \phi=18^{\circ}$ depends on the magnitude of the DC-component described by $A_{D C}$. Liu et.al. also reported a distortion of phase values during the transient state at the beginning of an $L T$ experiment [17]. Contrarily to the case presented in figure 4 they considered only two transients with different DC components. Breitenstein et.al. calculated even larger distortions of phase angle values for a case corresponding to $A_{D C}=$ $2 \mathrm{~K}[8]$.

It should be mentioned that this is the result of evaluating disharmonic functions with variables such as amplitude and phase that are only defined for harmonic functions. Thus, similar effects must be expected when other methods than FFT are exploited to evaluate phase differences in temperature transients of LT. This is of particular interest, if obtained values of phase differences are needed for the determination of material properties [7] or the depth of a defect [11]

\section{Measured temperature transients}

Following the theoretical consideration, temperature transients of real lock-in experiments are regarded. Only optically excited LT investigations are presented. The description of the experimental details is limited to what is necessary, since we only want to show which temperature transients can occur at all. 
Table 1. Overview of the $L T$ experiments presented below

\begin{tabular}{|c|c|c|c|c|}
\hline sample & Heating source & $\begin{array}{c}\text { Lock-in } \\
\text { frequency }\end{array}$ & $\begin{array}{c}\text { Number of } \\
\text { Lock-in periods }\end{array}$ & remarks \\
\hline $\begin{array}{l}\text { black PVC step wedge }(1 \ldots \\
8 \mathrm{~mm} \text { thick })\end{array}$ & 1 halogen lamp & $\begin{array}{c}2 \mathrm{~Hz} \\
0.01 \mathrm{~Hz}\end{array}$ & $\begin{array}{l}105 \\
16\end{array}$ & $\begin{array}{l}\text { periodic heating with square-wave pattern } \\
2 \mathrm{~Hz} \text { is quite fast for halogen lamps; thus the } \\
\text { amplitude of the AC component is reduced in } \\
\text { favour of the DC component }\end{array}$ \\
\hline $\begin{array}{l}\text { black-coated stainless-steel } \\
\text { plate ( } 6 \mathrm{~mm} \text { thick) }\end{array}$ & 2 halogen lamps & $2 \mathrm{~Hz}$ & 100 & $\begin{array}{l}\text { LWIR and MWIR camera were used in the } \\
\text { same setup }\end{array}$ \\
\hline $\begin{array}{l}\text { black-coated stainless-steel } \\
\text { plate ( } 4.5 \mathrm{~mm} \text { thick) }\end{array}$ & LED array & $\begin{array}{c}0.1 \mathrm{~Hz} \\
1 \mathrm{~Hz} \\
10 \mathrm{~Hz}\end{array}$ & $\begin{array}{l}10 \\
100 \\
1000\end{array}$ & \\
\hline $\begin{array}{l}\text { stainless-steel plate } \\
\text { (4.5 mm thick) }\end{array}$ & VCSEL-array & $1 \mathrm{~Hz}$ & 70 & Uncoated metal surface with high reflectivity \\
\hline
\end{tabular}

The first experiment was LT at a step wedge with $16 \mathrm{~cm} \times 8 \mathrm{~cm}$ size. It was made from black PVC with 8 steps and thicknesses between 1 and $8 \mathrm{~mm}$. Heating was performed with a halogen lamp equipped with a plexiglass filter to reduce direct reflections from the hot halogen lamp at the heated surface. The surface temperature was recorded by an MWIR camera with $200 \mathrm{~Hz}$ full frame rate, but reduced frame rates were used for low frequencies. Figure 5 is a difference thermogram of an experiment with $2 \mathrm{~Hz}$ lock-in frequency after $18 \mathrm{~s}$ resp. 36 periods. The thickness increases from left to right. At that time, only the thinnest steps with $1 \mathrm{~mm}$ and $2 \mathrm{~mm}$ can be distinguished from the other steps directly in the thermogram.

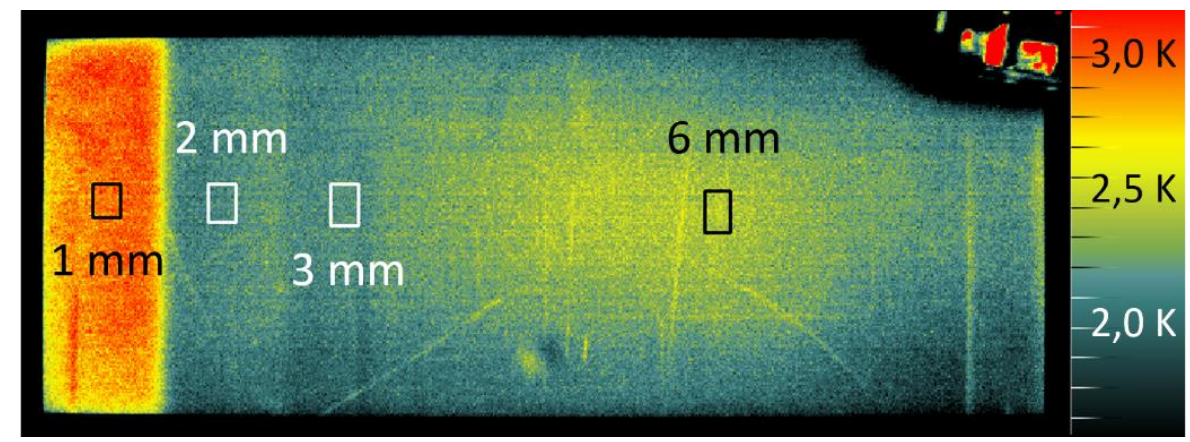

Fig. 5: Difference thermogram of a black PVC step wedge after 36 periods with $2 \mathrm{~Hz}$ lock-in frequency realized by illuminating with an oscillating halogen lamp, the warmer area in the middle is due the spatial power distribution of this setup, the rectangles mark the ROIs for the determination of the respective temperature transients

The next figure 6 comprises the temperature transients measured and spatially averaged within the rectangles shown in figure 5. The respective curves are not linear, but rather bend like a square root function as discussed in [3] for thermal thick probes at the beginning. The curves demonstrate also that the DC temperature increase is determined by the thickness or heat capacity as well as the intensity of the absorbed optical power. Thus, the temperature at 6 mm thickness is slightly larger than at $2 \mathrm{~mm}$ thickness after $10 \mathrm{~s}$ periodical heating (see insert in figure 5) due to the spatially inhomogeneous power density.

For comparison, figure 7 comprises different temperature transients at the same specimen as a result of a longterm experiment with $0.01 \mathrm{~Hz}$ lock-in frequency. The pointed shape at the maxima of the oscillations results from periodic heating with square-wave excitation instead of a sinusoidal excitation. However, the general shape of the DC component can be inspected clearly: the slope decreases until the DC-temperature reaches a constant final level. This is the optimum for LT because heat losses compensate the absorbed power and the DC component disappears completely. As mentioned before, reaching this equilibrium state requires very long observation periods. In the presented case the final state for the entire thickness range up to $8 \mathrm{~mm}$ was not reached within the 27 minutes long observation period. 


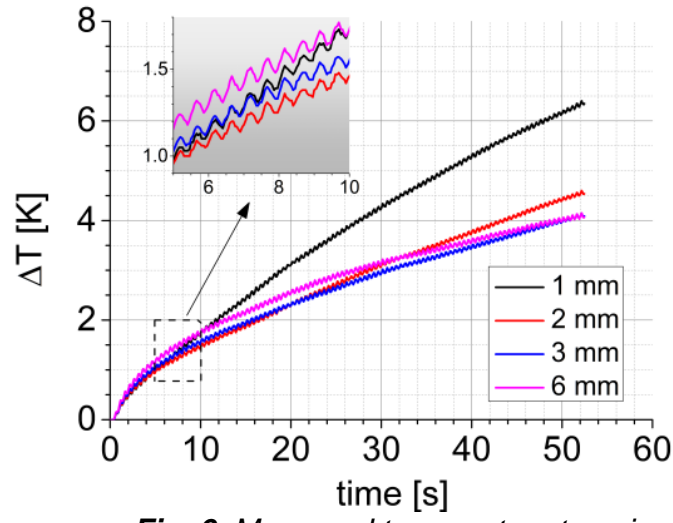

Fig. 6: Measured temperature transients at different thicknesses of a PVC step wedge during 105 lock-in periods of $2 \mathrm{~Hz}$ frequency, the insert is a magnification of the marked region to present the oscillating heating pattern

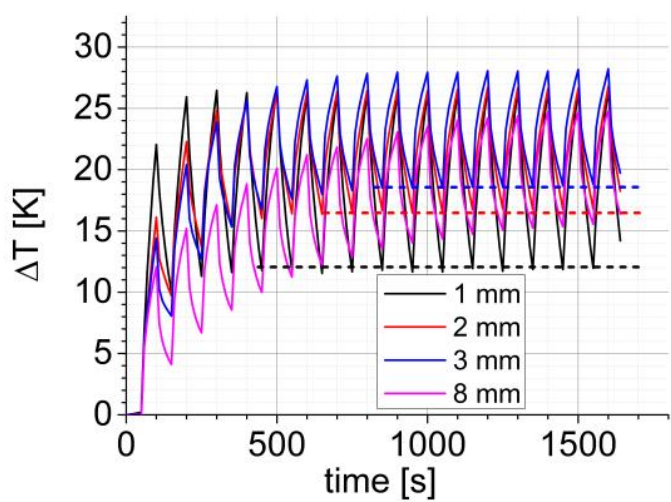

Fig. 7: Measured temperature transients at different thicknesses of a PVC step wedge during 16 lock-in periods of $0.01 \mathrm{~Hz}$ frequency, the dotted lines connecting the respective curve minima indicate that the final temperature has been reached for the corresponding thickness

The next LT experiment to be considered concerns a black coated stainless-steel plate (1.4034) of $6 \mathrm{~mm}$ thickness. Heating was performed with two halogen lamps of $1.2 \mathrm{~kW}$ power each (total irradiance at the sample is approximately $0.5 \mathrm{~W} / \mathrm{cm}^{2}$ ) and equipped with two sheets of glass to reduce direct reflections from the hot halogen lamps at the heated surface. Contrary to the experiment presented before the lock-in excitation was realized by real sinusoidal heating. The surface temperature was recorded by an MWIR camera with $200 \mathrm{~Hz}$ full frame rate as well as by an LWIR camera with $100 \mathrm{~Hz}$ full frame rate. Reduced frame rates were used for low frequencies. Figures 8 and 9 show the direct comparison of the transients recorded with the different cameras. The difference between both transients is striking.

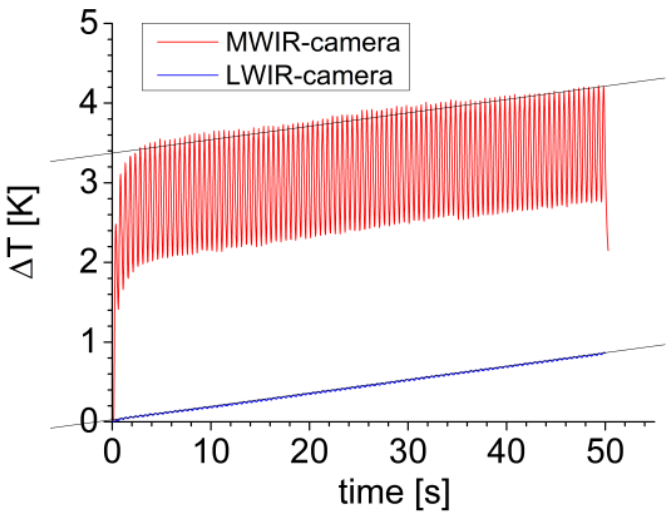

Fig. 8: Comparison of temperature transients recorded with an MWIR and an LWIR camera, measured at a $6 \mathrm{~mm}$ thick stainless-steel plate during 100 lock-in periods of $2 \mathrm{~Hz}$ frequency, the black lines are only guides for the eye

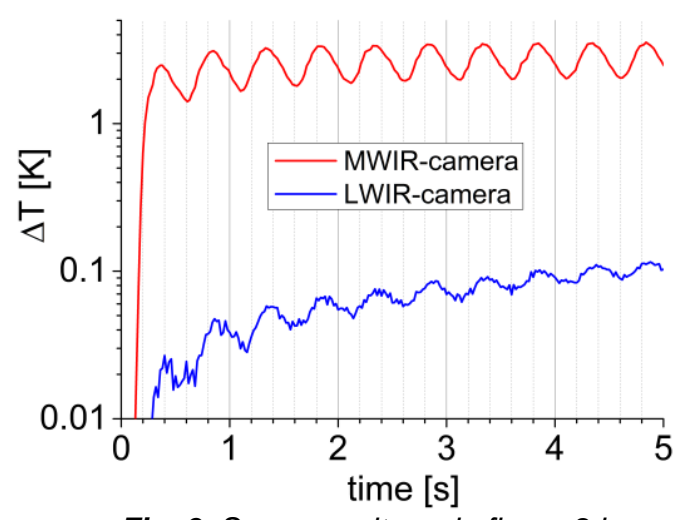

Fig. 9: Same results as in figure 8 in a semilog plot, where only the first 5 seconds are shown

While the LWIR camera observed a nearly perfect linear DC component, the MWIR camera observed a strong increase within the first periods. The amplitudes of temperature oscillations are much larger (almost two orders of magnitude). However, the slope of the DC component is nearly the same for both cameras after 10 periods. This observation can only be explained by strong residual reflections from the lamps at the specimen surface. The use of glass sheets was not sufficient to block these reflections in the spectral range where the MWIR camera is sensitive for. Here, the resulting reflection signal was much higher than the emission signal due to the real temperature of the surface. And the corresponding value for the phase lag or phase differences will be reduced (systematic error), because the reflected signal has a phase lag of zero. Vavilov reported a similar strong influence of direct reflections in case of pulsed thermography [18].

Further LT experiments were carried out using an LED-array with an irradiance of $0.15 \mathrm{~W} / \mathrm{cm}^{2}$ at a wavelength of $850 \mathrm{~nm}$ as oscillating heating source. Since there is no spectral overlap of the LED emission spectrum and the spectral sensitivity range of the MWIR camera oscillating reflections of the heating source can be neglected. More details can be found in [19]. The surface temperature was recorded by an MWIR camera with $200 \mathrm{~Hz}$ full frame rate, but reduced frame rates were used for low frequencies. The sample was a black coated stainless-steel plate (1.4301) of 4.5 mm thickness. 
Figure 10 displays three temperature transients corresponding to $0.1 \mathrm{~Hz}, 1 \mathrm{~Hz}$ and $10 \mathrm{~Hz}$ on the same scale. The DC components of all three curves have similar shapes and slopes, slight differences are not discussed here. The dotted straight line indicates that the DC components are not perfectly linear but slightly curved. The apparently strong temperature rise observed with the MWIR camera during heating with halogen lamps did not appear because the LED arrays do not warm up significantly during operation.

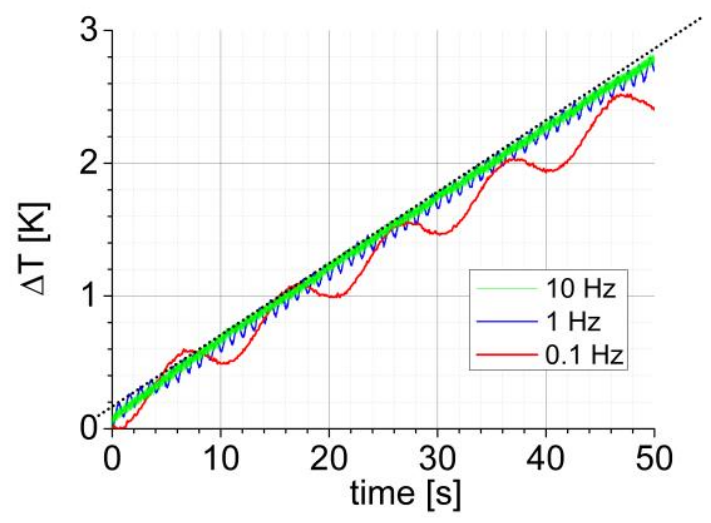

Fig. 10: Comparison of temperature transients at different lock-in frequencies, recorded with a MWIR camera, measured at a $4.5 \mathrm{~mm}$ thick stainless-steel plate during $50 \mathrm{~s}$, the black dotted line indicates a perfectly linear shape

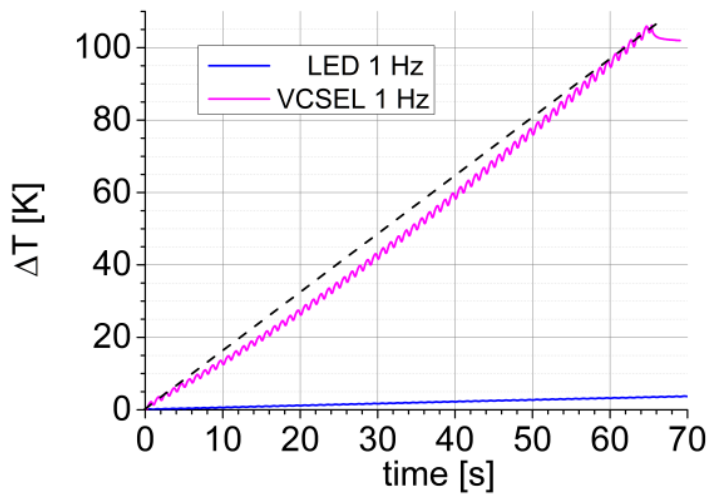

Fig. 11: Comparison of temperature transients generated by different heating sources both with $1 \mathrm{~Hz}$, recorded with a MWIR camera, measured at a $4.5 \mathrm{~mm}$ thick stainless-steel plate during $70 \mathrm{~s}$, the black dashed line indicates a perfectly linear shape

The last LT experiment to be considered was realized at the same specimen, here without black coating, but with an array of VCSEL diodes with an optical output power of $2.4 \mathrm{~kW}$ (irradiance of $19 \mathrm{~W} / \mathrm{cm}^{2}$ ) at a wavelength of $(980 \pm 10)$ $\mathrm{nm}$ [16]. The MWIR camera was also the same as in the case of the LED array. As to be expected, the VCSEL array generated a much stronger increase of the DC component due to its higher irradiance. In case of the VSCEL data the displayed temperatures correspond to an estimated emissivity of 0.2 for the metal surface.

Considering the respective curve shape of the surface temperature transient, the DC component rises stronger than linear. The reason for this effect is probably caused by the dynamic of the temperature control of the VCSEL array. The internal cooling circuit of the VCSEL array is coupled to a PID controlled water chiller.

The presented examples show a variety of possible curves for the temperature transients of the DC component in an LT experiment. The respective curve shape is determined by a series of experimental details:

i) Heat capacity of the specimen (thickness, density and specific heat capacity)

ii) Heat flux density generated by the heating source (optical output power, spectral characteristics in relation to the sensitivity of the used IR-camera, homogeneity of the irradiance, absorptance and reflectance of the illuminated sample surface)

iii) Heat loss processes convection, thermal radiation and perhaps heat dissipation via the mount (all heat losses are determined by the temperature gradient between specimen surface and surrounding).

The variety of parameters makes it practically impossible to predict the curve shape of the DC component before conducting an LT inspection. Therefore, a careful analysis of the respective temperature transients is essential to eliminate the DC component of the transient effectively, if it is intended.

\section{Phase angle differences at measured temperature transients}

In this section the disturbing influence of the DC component in LT temperature transients on the resulting phase angle difference is studied at one experimental data set. Here, the data of LT with the LED array at $0.1 \mathrm{~Hz}$ applied to the $4.5 \mathrm{~mm}$ thick stainless-steel plate were used (red curve in figure 10 corresponds to a reference region of $4.5 \mathrm{~mm}$ thickness). In order to investigate phase differences, temperature transients with phase lag in the AC component are required. The investigated specimen contains flat bottom holes with different diameters and depths (or remaining wall thicknesses) at the rear side. In the following, the temperature transients at the areas D1 and D2, which are above the $4 \mathrm{~mm}$ diameter holes, each with remaining wall thicknesses of $0.23 \mathrm{~mm}$ and $0.5 \mathrm{~mm}$, were selected for further evaluation. The phase angle difference is calculated as the difference between the phase values of regions D1 and D2 $\varphi_{D 1 / 2}$ with respect the phase values of a reference region (denoted Ref.) $\varphi_{\text {Ref }}$. 
Due to their almost linear curve shape (see figure 10) the DC components were reduced effectively by subtracting a simple linear fit of the respective temperature transients. Figure 12 displays the resulting difference curves (named as exp.). Additionally, these data sets were fitted by means of a sine function:

$$
S_{f i t}(t)=S_{0}+A_{0} \cdot \sin (2 \pi f \cdot t+\varphi)
$$

with $S_{0}, A_{0}$, and $\varphi$ as free fit parameters ( $f$ is given by the experiment as $0.1 \mathrm{~Hz}$ ). The fitted curves are also shown in figure 12 (named as fit). The deviation between the corrected experimental data and the fit with the sine function is small but detectable especially at the first minimum.

Figure 13 shows a part of the $6^{\text {th }}$ period of the corrected experimental data. The $6^{\text {th }}$ period was chosen because the linear correction worked quite well here. The idea of this figure is to enable a direct reading of phase differences in the diagram (graphical solution). It is focused at one maximum to recognize the exact time position and thus the phase angle relations. The straight lines in Figure 13 correspond to fits using Eq. (4), but, in contrast to the ones shown in Figure 12 , the data range was limited to only the $6^{\text {th }}$ period $50 \mathrm{~s}$ after the beginning. The arrows indicate the corresponding maxima and the related phase angle differences corresponding to the different steel thicknesses are evident.

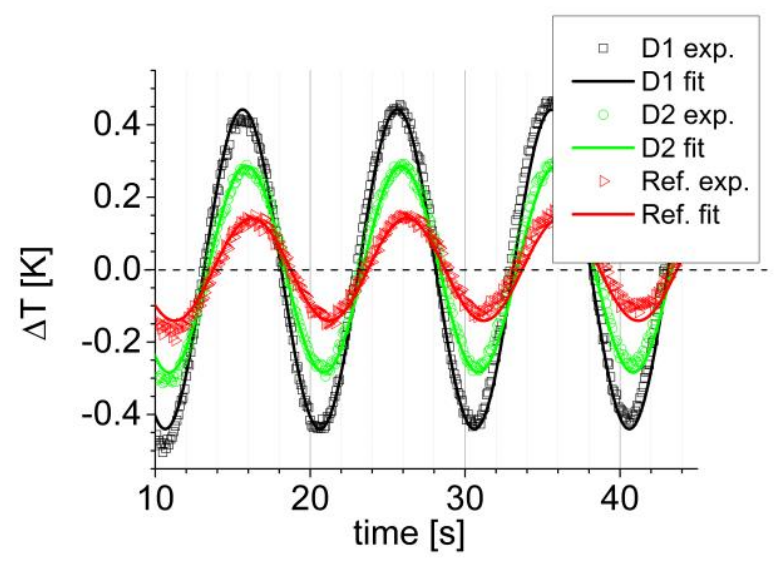

Fig. 12: Comparison of DC-corrected temperature transients of flat bottom holes with a diameter of $4 \mathrm{~mm}$ and $0.23 \mathrm{~mm}(D 1), 0.5 \mathrm{~mm}(D 2)$ remaining wall thickness and $4.5 \mathrm{~mm}$ thick stainless steel (Ref), fits were conducted by means of Eq. (4).

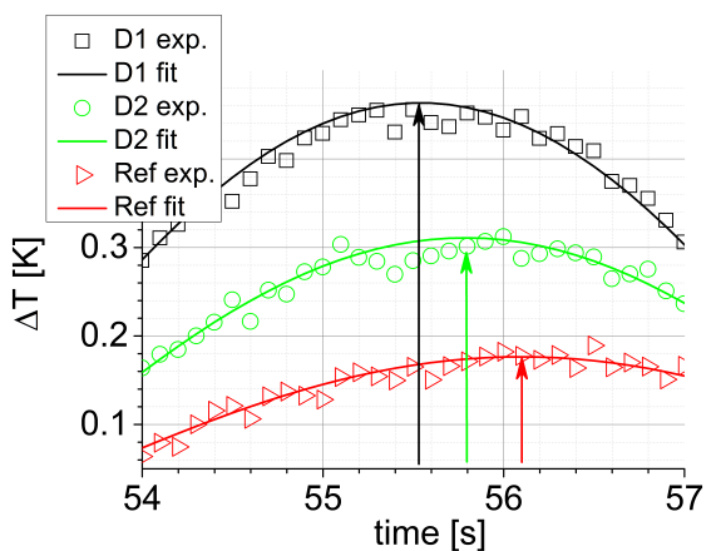

Fig. 13: Comparison of DC-corrected temperature transients of flat bottom holes with a diameter of $4 \mathrm{~mm}$ and $0.23 \mathrm{~mm}(D 1), 0.5 \mathrm{~mm}(D 2)$ remaining wall thickness and $4.5 \mathrm{~mm}$ thick stainless steel

(Ref), fits were conducted by means of Eq. (4), the arrows point to the respective curve maxima.

The resulting phase angle differences $\Delta \varphi_{1 / 2}=\varphi_{D 1 / 2}-\varphi_{\text {Ref }}$ are compiled in the following table 2 , together with values from other evaluations.

Table 2. Values of phase angle differences obtained at the same data set (includes 10 periods at $0.1 \mathrm{~Hz}$ lock-in excitation by means of an LED array at stainless steel, temperature difference transients at 3 ROIs)

\begin{tabular}{|l|c|c|c|}
\hline \multicolumn{1}{|c|}{ Data processing } & $\Delta \varphi_{1}$ (D1-Ref) & $\Delta \varphi_{2}$ (D2-Ref) & Comments \\
\hline $\begin{array}{l}\text { IR camera software module (IRBIS prof. V. 152 } \\
\text { from Infratec). Offline Lock-In calculation at } \\
\text { uncorrected data, 10 periods }\end{array}$ & $29.5^{\circ}$ & $16.7^{\circ}$ & $\begin{array}{l}\text { Seems to use only uncorrec- } \\
\text { ted data }\end{array}$ \\
\hline $\begin{array}{l}\text { FFT with ORIGIN at uncorrected data (AC+DC } \\
\text { parts), 10 periods }\end{array}$ & $29.63^{\circ}$ & $16.76^{\circ}$ & \\
\hline $\begin{array}{l}\text { FFT with ORIGIN at corrected data (only AC } \\
\text { part),10 periods }\end{array}$ & $26.72^{\circ}$ & $11.66^{\circ}$ & \\
\hline $\begin{array}{l}\text { Sine fit with ORIGIN of the corrected data (only } \\
\text { AC part), only the 6 }\end{array}$ & $20.04^{\circ}$ period & $10.98^{\circ}$ & \\
\hline $\begin{array}{l}\text { Visual inspection of the time lag in the corrected } \\
\text { data (only AC part), only the 6 }\end{array}$ & $19.8^{\circ}$ period & $9.0^{\circ}$ & Calculated from time lags \\
\hline
\end{tabular}


In the considered case the true values of the phase difference are not known (contrary to the simulated transients), thus only the obtained differences are regarded. In comparison to the phase differences obtained from uncorrected data, a deviation of about $9.5^{\circ}$ occurred. It is interesting to compare this value with the simulated data. The comparison requires values of $A_{D C}$ and $A_{A C}$ for uncorrected experimental transients. For D1 the following numbers were found:

1. slope of the linear correction fit for D1 data set (red curve in figure 10) $m=0.0463 \mathrm{~K} / \mathrm{s}$, this corresponds to $m_{10}=$ $0.463 \mathrm{~K}$ within one period at $0.1 \mathrm{~Hz}$

2. $A_{0}=0.44 \mathrm{~K}$ (fitted amplitude of the black curve in figure 12 according to Eq. 4)

Now, it must be regarded, that $A_{D C}$ used in figures 1-4 was originally related to $A_{A C}=1 \mathrm{~K}$. In order to evaluate a suited comparable value for $A_{D C}$ of the experimental data, the value of $m_{10}$ has to be normalized to obtain the correct value of $A_{D C}$.

$$
A_{D C}=m_{10} / A_{0}=1.05(\text { in } \mathrm{K})
$$

The curve in figure 4 indicates a phase error of $8^{\circ}$ for $A_{D C}=1 \mathrm{~K}$. The difference between uncorrected data (second row in table 2) and corrected data ( $4^{\text {th }}$ row in table 2) for the phase difference is $9.6^{\circ}$ and thus in the same order of magnitude as predicted by the simulation. It has to be noted that the simulation was done for $50 \mathrm{mHz}$ only and the influence of the frequency on the magnitude of the phase error has not yet been investigated. At this point it can be concluded that eliminating the DC component of a temperature transient in an LT experiment leads to reduced values for the phase differences and the same effect was found at simulated temperature transients with known phase lags. This finding confirms Breitenstein's et.al. comments on this issue [8]. Their proposed compensation of the DC component by means of a linear regression of the initial and the final temperature alone has some drawbacks concerning the signal to noise ratio of the evaluated phase values. In view of the drastically increased computer capacities available on site, precise analytical compensation of the DC component can easily be realized even with standard programs, whereas a simplified data processing as proposed in 2003 is therefore no longer necessary. It is not the intention of the paper to discuss this point in order to identify the most suited data evaluation procedure, but the suppression of the DC component of the transients seems to be essential to obtain correct data for phase differences.

\section{Conclusions}

LT is based on a periodic heating of a sample. The underlying theory of thermal waves is based on a purely harmonic heating that is difficult to achieve in practice. This would require a change between heating and cooling, which is virtually impossible to achieve experimentally if optical heat sources are used. This paper describes the influence of nonharmonic heating on the phase lag of temperature oscillations at the sample surface. This study includes theoretical considerations with simulated curves as well as data comparison of different LT experiments. The simulations prove a systematic falsification of the phase values with increasing deviation from the ideal sinusoidal shape. To quantify the effect, the superposition of a sine function with a linearly increasing function was considered, whereby the slope was varied. The ratio of this slope to the amplitude of the sine function determines the magnitude of the phase error. Similar results were obtained by other authors.

The consideration of the raw data of different LT investigations showed a variety of possible shapes of curves. In particular, the spectral range of the optical radiation source must be completely separated from the sensitivity range of the IR camera to avoid interference. An almost linear temperature increase was observed only in a few cases.

The correction of a selected data set by subtracting a regression line led to a significant effect in the determination of phase differences obtained at low material thicknesses. The value of $29.6^{\circ}$ found on uncorrected data decreased to about $20^{\circ}$ after correction. The magnitude of this effect corresponded to the result with the simulated curves.

The question of an optimal length of the LT experiment must therefore remain unanswered, especially for more curved temperature transients. This requires a systematic investigation with simulated curved transients. However, the results obtained so far suggest that an LT measurement with numerous periods is probably not necessary if the DC component can be eliminated. And the expected reduction of $\mathrm{S} / \mathrm{N}$ with rising number of periods can only be achieved, if the correction of the DC component is sufficient. On the other hand, it is remarkable that none of the experiments presented in this study achieved in fact a thermal balance between warming and heat loss, which is usually preferred. In the case of optical sources with very high irradiance like lasers, it is practically impossible to achieve this thermal equilibrium. Therefore, errors in the determination of phase differences would be expected in any case if the DC component was not corrected.

\section{Acknowledgements}

The presented results have partially been achieved within the research project Development of standards for active thermography with lock-in excitation (BMWi 01FS12011). This project was supported by the Federal Ministry for Economic Affairs and Energy of Germany within the research programme Transfer of research and development results 
through standardisation. A special thank goes to Mathias Röllig from our department for the careful experiments with the different IR cameras.

\section{REFERENCES}

[1] Busse G., Wu D., Karpen W., "Thermal wave imaging with phase sensitive modulated thermography", Journal of Applied Physics, vol. 71(8), pp. 3962, 1992.

[2] Almond D.P., Patel P.M., "Photothermal Science and Techniques", London: Chapman \& Hall; 1996.241 p.

[3] Breitenstein O., Warta W., "Langenkamp M. Lock-in Thermography Basic and Use for Evaluating Electronic Devices and Materials", 2. ed, Springer, Berlin; 2010.

[4] Wallbrink C., Wade S.A., Jones R., "The effect of size on the quantitative estimation of defect depth in steel structures using lock-in thermography", Journal of Applied Physics, vol. 101(10), pp. 104907, 2007.

[5] Mandelis A., "Diffusion-wave fields Mathematicla Methods and Green Functions", Springer, New York 2001.

[6] Bai W., Wong B.S., "Photothermal models for lock-in thermographic evaluation of plates with finite thickness under convection conditions", Journal of Applied Physics, vol. 89(6), pp. 3275, 2001.

[7] Nolte P.W., Malvisalo T., Wagner F., Schweizer S., "Thermal diffusivity of metals determined by lock-in thermography", Quantitative InfraRed Thermography Journal, vol. 14(2), pp. 218-225, 2017.

[8] Breitenstein O., Langenkamp M. Lockin Thermography - Basics and Use for Functional Diagnostics of Electronic components. first ed., Springer, Berlin; 2003.

[9] Pickering S., Almond D., "Matched excitation energy comparison of the pulse and lock-in thermography NDE techniques", NDT\&E International, vol. 41(7), pp. 501-509, 2008.

[10] Meola C., Carlomagno G.M., "Impact damage in GFRP New insights with infrared thermography", Composites Part A: Applied Science and Manufacturing, vol. 41(12), pp. 1839-1847, 2010.

[11] Montanini R., Freni F., "Non-destructive evaluation of thick glass fiber-reinforced composites by means of optically excited lock-in thermography", Composites Part A: Applied Science and Manufacturing, vol. 43(11), pp. 2075-2082, 2012.

[12] Delanthabettu S., Menaka M., Venkatraman B., Raj B., "Defect depth quantification using lock-in thermography", Quantitative InfraRed Thermography Journal, vol. 12(1), pp. 37-52, 2015.

[13] Zhao H.X., Zhou Z.G., Fan J., Li G., Sun G.K., "Application of lock-in thermography for the inspection of disbonds in titanium alloy honeycomb sandwich structure", Infrared Physics \& Technology, vol. 81, pp. 69-78, 2017.

[14] Peng D., Jones R., "Lock-in thermographic inspection of squats on rail steel head", Infrared Physics \& Technology, vol. 57, pp. 89-95, 2013.

[15] Rajic N., Antolis C., "An investigation of noise performance in optical lock-in thermography", Infrared Physics \& Technology, vol. 87, pp. 1-10, 2017.

[16] Ziegler M., Thiel E., Studemund T., "Thermography using a 1D laser array - From planar to structured heating", Materials Testing, vol., pp., 2018 (in press).

[17] Liu W., Tang B., Jiang Y., "Status and problems of wind turbine structural health monitoring techniques in China", Renewable Energy, vol. 35(7), pp. 1414-1418, 2010.

[18] Vavilov V., "Noise-limited thermal/infrared nondestructive testing", NDT\&E International, vol. 61, pp. 16-23, 2014.

[19] Krankenhagen R., Altenburg S.J., "Direct comparison of two pyrometers and a low-cost thermographic camera for time resolved LWIR temperature measurements", Thermosense: Thermal Infrared Applications XXXIX, vol. 10214, pp., 2017. 